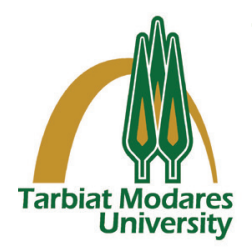

\title{
Risk Factors of Mortality among Hospitalized COVID-19 Patients in A Large University Hospital in Tehran, Iran
}

\section{A R T I C L E I N F O}

Article Type

Original Research

Authors

Mehrdad Haghighi, $M D^{1}$

Hossein Hatami, $M D-M P H^{2}$

Shayan Aryannezhad, $M D-M P H^{3}$

Negar Delbari, $M D-M P H^{3 *}$

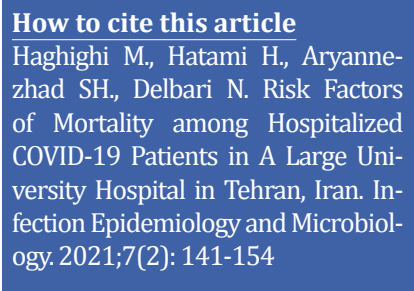

${ }^{1}$ Department of Infectious Diseases, Imam Hossein Teaching and Medical Hospital, Shahid Beheshti University of Medical Sciences, Tehran, Iran. ${ }^{2}$ Department of Public Health, School of Public Health and Safety and Environmental and Occupational Hazards Control Research Center, Shahid Beheshti University of Medical Sciences, Tehran, Iran. ${ }^{3}$ School of Public Health and Safety, Shahid Beheshti University of Medical Sciences, Tehran, Iran.

\footnotetext{
* Correspondence

Address: School of Public Health and Safety, Shahid Beheshti University of Medical Sciences, Tehran, Iran. Tel: +989196090911 ne.delbari@sbmu.ac.ir
}

\section{A B S T R A C T}

Backgrounds: Coronavirus disease 2019 (COVID-19) pandemic is considered as a global health challenge, and Iran is among the top ten countries with the highest mortality rate. This study aimed to analyze epidemiological, clinical, and laboratory features of hospitalized SARS-CoV-2 infected patients and identify the risk factors of mortality among them to facilitate the prioritization of resources and vaccine allocation in the population using data available in a large university hospital in Tehran, Iran.

Materials \& Methods: From February 20 to May 14, 2020, a total of 684 COVID-19 confirmed cases were admitted to Imam Hossein University hospital in Tehran and enrolled in this retrospective observational study.

Findings: The mean age of the participants was $59.48 \pm 16.65$ years, and $55.8 \%$ were male. Compared to survivors, non-survivors $(n=127,18.57 \%)$ were older $(69.09 \pm 14.16$ vs. $57.29 \pm 16.40$ ), had higher average number of comorbidities, and more frequently suffered from dyspnea, convulsion, loss of consciousness (LOC), tachycardia, tachypnea, and hypoxia. They also had higher levels of leukocytes, neutrophil count, C-reactive protein (CRP), creatine phosphokinase (CPK), aspartate aminotransferase (AST), lactate dehydrogenase (LDH), international normalized ratio (INR), prothrombin time (PT), partial thromboplastin time (PTT), urea, and serum creatinine $(p<.05)$. Serum potassium (K), lymphocyte count, hemoglobin, $\mathrm{HCO}_{3}$, and serum sodium $(\mathrm{Na})$ were significantly lower in non-survivors. Multivariable logistic regression analysis results revealed that nine variables were positively associated with mortality.

Conclusion: Among COVID-19 patients admitted to the hospital, having more than two or three comorbidities, loss of consciousness, SpO2<90\%, increased CRP, CPK, urea, and serum potassium, were associated with mortality.

\section{Keywords: SARS-CoV-2, COVID-19, Risk factors, Mortality.}

\section{CITATION LINKS}

[1] Li Q, Guan X, Wu P, Wang X, Zhou L, Tong Y, et al. Early ... [2] World Health Organization. WHO ... [3] Struyf T, Deeks JJ, Dinnes J, Takwoingi Y, Davenport C, Leeflang MM, et al. Signs ... [4] Mahumud RA, Kamara JK, Renzaho AMN. The epidemiological ... [5] Abdi M. Coronavirus disease 2019 ... [6] Iran Population 2020. World ... [7] Arab-Zozani M, Ghoddoosi-Nejad D. Covid-19 in ... [8] COVID-19 coronavirus pandamic. Worldometer ... [9] Javanian M, Bayani M, Shokri M, ... [10] Shahriarirad R, Khodamoradi Z, Erfani A, Hosseinpour H, Ranjbar K, Emami Y, et al. Epidemiological ... [11] Akbari A, Emami A, Javanmardi F, Pirbonyeh N, Fadakar N. Early ... [12] WHO COVID-19 Case definition. WHO website; 2020 ... [13] Hopkins J. Mortality ... [14] Jalili M, Payandemehr P, Saghaei A, Nouri Sari H, Safikhani HR, Kolivand P. Characteristics ... [15] Beeching NJ, Fletcher TE, Fowler R. BMJ best ... [16] Bchetnia M, Girard C, Duchaine C, Laprise C. The outbreak of ... [17] Thoguluva Chandrasekar V, Venkatesalu B. Systematic review and ... [18] Dong Y, Dai T, Wei Y, Zhang L, Zheng M, Zhou F. A systematic ... [19] Wiersinga WJ, Rhodes A, Cheng AC, Peacock SJ, Prescott HC. Pathophysiology ... [20] Richardson S, Hirsch JS, Narasimhan M, Crawford JM, McGinn T, Davidson KW, et al. Presenting ... [21] Jin J-M, Bai P, He W, Wu F, Liu X-F, Han D-M, et al. Gender ... [22] Sharma G, Volgman AS, Michos ED. Sex ... [23] Parohan M, Yaghoubi S, Seraji A, Javanbakht MH, Sarraf P, Djalali M. Risk ... [24] Wu C, Chen X, Cai Y, Xia Ja, Zhou X, Xu S, et al. Risk .... [25] Wee LE, Chan YFZ, Teo NWY, Cherng BPZ, Thien SY, Wong ... [26] Spinato G, Fabbris C, Polesel J, Cazzador D, Borsetto D, Hopkins ... [27] Ellul MA, Benjamin L, Singh B, Lant S, Michael BD, Easton A, et al. Neurological ... [28] Paterson RW, Brown RL, Benjamin L, Nortley R, Wiethoff S, Bharucha T, et al. The ... [29] Cascella M, Rajnik M, Cuomo A, Dulebohn SC, Di Napoli R. Features ... [30] Rentsch CT, Kidwai-Khan F, Tate JP, Park LS, ... [31] Pettirosso E, Giles M, Cole S, Rees M. COVID-19 and ... [32] Caussy C, Wallet F, Laville M, Disse ... [33] Simonnet A, Chetboun M, Poissy J, Raverdy V. High ... [34] Dietz W, Santos-Burgoa C. Obesity and ... [35] Onder G, Rezza G, Brusaferro S. Case-fatality rate and ... [36] Beeching NJ, Fletcher TE, Fowler R. BMJ best practice - Coronavirus ... [37] Lippi G, South AM, Henry BM. Electrolyte ... [38] Tavakolpour S, Rakhshandehroo T, Wei EX, Rashidian M. Lymphopenia ... [39] Tan L, Wang Q, Zhang D, Ding J, Huang Q Tang Y-Q et al. Lymphopenia ... 


\section{Introduction}

In late December 2019, a series of pneumonia cases, caused by severe acute respiratory syndrome coronavirus 2 (SARS-CoV-2), were reported in Wuhan, China ${ }^{[1]}$. The novel disease was later called coronavirus disease 2019 (COVID-19), rapidly spread throughout the world, and was announced as a global pandemic by the World Health Organization (WHO) in mid-March 2020. COVID-19 is currently considered as a global health crisis, affecting nearly all countries with more than 102.0 million infected patients and 2.2 million deaths worldwide by the end of January $2021^{\text {[2]. }}$

Clinical manifestations of SARS-CoV-2 infection are very diverse; whilst some patients might be asymptomatic, many have mild to moderate symptoms (i.e. cough, fever, myalgia, fatigue, and headache), and few have more severe signs and symptoms (i.e. breathlessness and increased respiratory rate) with higher susceptibility to critical conditions ${ }^{[3]}$. Therefore, given the wide range of clinical manifestations and outcomes of the disease, a better understanding of the risk factors associated with serious events and mortality could be a crucial step in tackling this pandemic. The presence of comorbidities such as hypertension, diabetes mellitus, cardiovascular diseases, and respiratory diseases have been identified as dominant indicators of mortality and poor outcomes among patients [4].

As one of the first countries experiencing COVID-19 outbreak, Iran is currently among the top ten countries with the highest mortality rate, it is still dealing with this growing epidemic ${ }^{[2]}$ and considered as an important focal point for the disease spread in the Middle East and even in the world [5]. Moreover, compared to china and other developed countries, which are currently the main sources of available knowledge regarding COVID-19 in the literature, Iran is different in many aspects, such as having a lower detection rate, higher case fatality rate, inadequate number of hospital beds per capita, and distinctive population pyramid [6-8]. Therefore, it is essential to address the characteristics of COVID-19 and infected population in this country with limited resource to better manage intensive care unit (ICU) capacity as well as better prioritize vaccine allocation. However, studies assessing epidemiological aspects of COVID-19 in Iran are few and mainly narrow-scoped based on small sample sizes with limited generalizability to the whole population [9-11].

Objectives: This study aimed to analyze demographic, epidemiological, clinical, and laboratory features of hospitalized SARSCoV-2 infected patients admitted to a large referral hospital in Tehran, Iran and evaluate the baseline risk factors associated with mortality outcome.

\section{Materials and Methods}

Study participants: Imam Hossein University hospital was allocated to provide medical services for patients with COVID-19 infection from February 2020 in Tehran, Iran. Among patients referring to the hospital, a total of 3334 suspected and probable SARSCoV-2 cases were admitted based on clinical judgment from February 20 (admission date of the first SARS-CoV-2 confirmed case) to May 14, 2020. After excluding those with negative results in real-time reverse transcription polymerase chain reaction (RT-PCR) tests $(n=2643)$ and those aged less than 18 years $(n=7)$, a total of 684 adults were diagnosed with SARS-CoV-2 infection using RT-PCR of nasopharyngeal specimens and included in the present retrospective observational study.

Data collection: Patients' medical records including epidemiological and demographic data, clinical records, including symptoms 
and signs at admission time, underlying comorbidities, and the need for invasive (IMV) or non-invasive mechanical ventilation (NIMV), as well as outcome data such as discharge/mortality and laboratory test results were retrospectively reviewed by two trained physicians.

Demographic data and information about patients' weight and height were recorded in the triage. Patients' data about the history of previous diseases and physical examinations including vital signs and pulse oximetry were recorded by trained physicians and nurses at the beginning in the hospital emergency department (ED). The first blood samples were taken from patients at admission time and sent to the hospital laboratory. Nasopharyngeal swabs specimens were collected from suspected and probable cases, sent to the laboratory, and tested using RT-PCR assays based on WHO protocols.

Definitions: In the present study, suspected and probable cases of SARS-CoV-2 infection were defined according to WHO COVID-19 case definition ${ }^{[12]}$. Confirmed cases were also defined based on WHO definition as a person with at least one positive SARS-CoV-2 RT-PCR result. Participants were divided based on their outcomes into survivors (those discharged successfully) and nonsurvivors (those with all-cause mortality during hospitalization). The length of hospital stay was calculated as discharge date minus admission date. Body mass index (BMI) was calculated by dividing weight in kilograms by height in meters squared (kg/ $\mathrm{m}^{2}$ ). Neutrophil and lymphocyte counts were calculated by multiplying the percentages of neutrophils and lymphocytes by leukocytes count, respectively.

Statistical analysis: All statistical analyses were performed using SPSS software Ver. 19.0, and a 2-tailed $p$ value less than .05 was considered as statistically significant.
Quantitative variables were compared between the two survivors and nonsurvivors groups using independent t-test for normally distributed variables and MannWhitney U test for non-normally distributed variables and presented as mean \pm SD or median as well as interquartile range (IQR), respectively. Categorical variables were compared with chi-square or Fisher exact 2-tailed tests and presented as percentages. All categorical variables significantly different between the two groups were included in univariate logistic regression analysis, and then variables with significant odds ratios for mortality in univariate models were included simultaneously in multivariate logistic regression analysis for determining the risk factors associated with adverse outcomes.

Approval: The present study was approved by Iran National Committee for Ethics in Biomedical Research with the ethical code number: IR.SBMU.PHNS.REC.1399.030. Informed consents were obtained from the study participants, and their privacy was maintained during the study.

\section{Findings}

Demographic and epidemiological characteristics: Among 3334 patients admitted to Imam Hossein University hospital from February 20 to May 14, 2020, a total of 684 SARS-CoV-2 confirmed patients $(20.52 \%)$ with the age range of more than 18 years were included in this analysis. Patients' epidemiological and demographic data as well as data about their outcomes in both groups of survivors $(n=557)$ and non-survivors $(n=127,18.57 \%)$ are shown in Table 1. The mean age of the patients was $59.48 \pm 16.65$ years. There was a significant difference between the two groups in terms of age $(57.29 \pm 16.40$ in survivors, $69.09 \pm 14.16$ in non-survivors, $p<.001)$; most patients were $40-65$ years old (46.1\%) in total and 
Table 1) Demographic and epidemiological characteristics of hospitalized COVID-19 patients.

\begin{tabular}{|c|c|c|c|c|}
\hline & $\begin{array}{l}\text { All Patients } \\
\quad(n=684)\end{array}$ & $\begin{array}{c}\text { Survivors } \\
(n=557)\end{array}$ & $\begin{array}{l}\text { Non-Survivors } \\
(n=127)\end{array}$ & P-Value \\
\hline \multicolumn{5}{|l|}{ Gender } \\
\hline Male & $382(55.8 \%)$ & 305 (54.8\%) & $77(60.6 \%)$ & \multirow{2}{*}{.229} \\
\hline Female & $302(44.2 \%)$ & $252(45.2 \%)$ & $50(39.4 \%)$ & \\
\hline Age (years) & $59.48 \pm 16.65$ & $57.29 \pm 16.40$ & $69.09 \pm 14.16$ & $<.001$ \\
\hline$<40$ & $94(13.7 \%)$ & $90(16.2 \%)$ & $4(3.1 \%)$ & \multirow{3}{*}{$<.001$} \\
\hline $40-65$ & $315(46.1 \%)$ & $275(49.4 \%)$ & $40(31.5 \%)$ & \\
\hline$\geq 65$ & $275(40.2)$ & $192(34.5 \%)$ & $83(65.4 \%)$ & \\
\hline BMI $\left(\mathrm{kg} / \mathrm{m}^{2}\right)$ & $27.59 \pm 5.48$ & $27.72 \pm 5.61$ & $27.04 \pm 4.85$ & .385 \\
\hline \multicolumn{5}{|l|}{ Comorbidities } \\
\hline Diabetes mellitus & $182(27.5 \%)$ & $138(25.7 \%)$ & $44(34.9 \%)$ & .037 \\
\hline Hypertension & $222(33.5 \%)$ & $164(24.8 \%)$ & $58(46.0 \%)$ & .001 \\
\hline CVD & $130(19.6 \%)$ & $86(16.0 \%)$ & $44(34.9 \%)$ & $<.001$ \\
\hline CKD & $48(7.3 \%)$ & $35(6.5 \%)$ & $13(10.3 \%)$ & .140 \\
\hline COPD & $13(2.0 \%)$ & $10(1.9 \%)$ & $3(2.4 \%)$ & .721 \\
\hline Asthma & $26(3.9 \%)$ & $21(3.9 \%)$ & $5(4.0 \%)$ & 1.000 \\
\hline Malignancy & $57(8.6 \%)$ & $49(9.1 \%)$ & $8(6.3 \%)$ & .325 \\
\hline Immunosuppression & $59(8.9 \%)$ & $51(9.5 \%)$ & $8(6.3 \%)$ & .269 \\
\hline CVA & $31(4.7 \%)$ & $21(3.9 \%)$ & $10(7.9 \%)$ & .057 \\
\hline Obesity & $92(28.0 \%)$ & $78(29.1 \%)$ & $14(23.0 \%)$ & .334 \\
\hline Pregnancy & $10(1.5 \%)$ & $10(1.9 \%)$ & $0(0 \%)$ & .222 \\
\hline $\begin{array}{l}\text { Numbers of } \\
\text { comorbidities }\end{array}$ & $1.29 \pm 1.23$ & $1.20 \pm 1.22$ & $1.63 \pm 1.21$ & $<.001$ \\
\hline 0 & $223(32.6 \%)$ & $199(35.7 \%)$ & $24(18.9 \%)$ & \multirow{4}{*}{.002} \\
\hline 1 & $185(27.0 \%)$ & $148(26.6 \%)$ & 37 (29.1\%) & \\
\hline 2 & $153(22.4 \%)$ & $115(20.6 \%)$ & $38(29.9 \%)$ & \\
\hline 3 and more & $123(18.0 \%)$ & 95 (17.1\%) & $28(22.0 \%)$ & \\
\hline
\end{tabular}

Values are expressed as mean \pm SD or numbers (\%).

BMI: Body mass index, CVD: Cardiovascular disease, CKD: Chronic kidney disease, COPD: Chronic obstructive pulmonary disease, CVA: Cerebrovascular accident. 
in survivors group (49.4\%), whereas in nonsurvivors group, most patients were older than 65 years $(65.4 \%)$. The number of men was higher than the number of women in total (55.8\% men, $44.2 \%$ women); however, no significant difference was found in terms of gender between the two groups. The average BMI of patients was $27.59 \pm 5.48$, and there was no significant difference regarding BMI and obesity prevalence between the two groups. The average number of comorbidities was significantly higher in non-survivors compared with survivors $(1.63 \pm 1.21,1.20 \pm 1.22, p<.001)$, and $67.4 \%$ of patients had at least one underlying comorbidity. HTN (hypertension) $(n=222$, $33.5 \%)$, DM (diabetes mellitus) $(\mathrm{n}=182$, 27.5\%), and CVD (cardiovascular disease) $(n=130,19.6 \%)$ were the three most frequent comorbidities among patients, which were also significantly more prevalent among non-survivors than survivors. However, the present study found no significant differences between survivors and nonsurvivors regarding other comorbidities such as immunosuppression (8.9\%), malignancy $(8.6 \%)$, CKD (chronic kidney disease) (7.3\%), CVA (cerebrovascular accident) (4.7\%), asthma (3.9\%), and COPD (chronic obstructive pulmonary disease) $(2.0 \%)$. There were also 10 pregnant women in this study, all of whom were included in the survivors group.

Clinical features: Patients' clinical characteristics including symptoms and signs at admission time are depicted in Table 2 . The most common symptoms were cough $(\mathrm{n}=415,62.6 \%)$, fever $(\mathrm{n}=370,55.9 \%)$, and dyspnea ( $\mathrm{n}=349,52.65 \%)$, among which only dyspnea $(63.5 \%$ vs. $50.0 \%, p=.006)$, convulsion ( $4.8 \%$ vs. $0.7 \%, p=.005)$, and LOC (loss of consciousness) (19.7\% vs. $4.5 \%, p<.001$ ) were significantly higher in non-survivors group. However, myalgia $(45.3 \%$ vs. $34.9 \%, p=.035)$ was more prevalent among survivors compared with non-survivors. Vital signs associated with mortality at admission time were tachycardia (pulse rate $>100 / \mathrm{min}$ ) (26.0\% vs. $13.1 \%$, $p<.001$ ) and tachypnea (respiratory rate > $20 / \mathrm{min}$ ) (33.3\% vs. $17.7 \%, p<.001$ ), which were more common in non-survivors. Moreover, the average pulse rate $(94.3 \pm 20.2$, $p=.001)$ and respiratory rate $(20.67 \pm 5.5$, $p=.02$ ) were also higher among nonsurvivors. Also, $\mathrm{O}_{2}$ saturation assessed by pulse oximetry (SpO2) at admission time was also significantly different among survivors $(91.36 \pm 5.46)$ and no-survivors (83.87 \pm 10.44$) \quad(p<.001)$. Moreover, the prevalence of patients with $\mathrm{SpO} 2$ less than 90 was higher in non-survivors compared to survivors $(66.7 \%$ vs. $25.4 \%, p<.001)$. At admission time, fever $\left(\mathrm{T} \geq 38.5^{\circ} \mathrm{C}\right)$ was detected only in $17.0 \%(n=108)$ of patients, and there was no significant difference in average temperature and fever between the two groups. Moreover, no significant difference in mean diastolic and systolic BP was found between the two groups.

Laboratory findings: Patients' laboratory test findings are shown in Table 3. Compared with survivors, non-survivors had significantly higher levels of leukocytes, neutrophil count, CRP (C-reactive protein), CPK (creatine phosphokinase), AST (aspartate aminotransferase), LDH (lactate dehydrogenase), INR (international normalized ratio), PT (prothrombin time), PTT (partial thromboplastin time), urea, and serum creatinine $(p<.001)$. Moreover, serum potassium $(\mathrm{K})$, lymphocyte count, hemoglobin, $\mathrm{HCO}_{3}$, and serum sodium ( $\mathrm{Na}$ ) were significantly lower in non-survivors compared with survivors. The present study found no significant difference in terms of platelets, ESR (erythrocyte sedimentation rate), $\mathrm{PH}, \mathrm{PCO}_{2}$ (partial pressure of carbon dioxide), and ALT (alanine aminotransferase) levels between the two groups; however, the 
Table 2) Clinical characteristics of hospitalized COVID-19 patients.

\begin{tabular}{|c|c|c|c|c|}
\hline & $\begin{array}{l}\text { All Patients } \\
(n=684)\end{array}$ & $\begin{array}{c}\text { Survivors } \\
(n=557)\end{array}$ & $\begin{array}{c}\text { Non-Survivors } \\
(n=127)\end{array}$ & P-Value \\
\hline \multicolumn{5}{|l|}{ Symptoms } \\
\hline Cough & $415(62.6 \%)$ & $341(63.5 \%)$ & $74(58.7 \%)$ & .319 \\
\hline Fever & $370(55.9 \%)$ & $302(56.3 \%)$ & $68(54.0 \%)$ & .629 \\
\hline Dyspnea & $349(52.65 \%)$ & $269(50.0 \%)$ & $80(63.5 \%)$ & .006 \\
\hline Myalgia & $287(43.3 \%)$ & $243(45.3 \%)$ & $44(34.9 \%)$ & .035 \\
\hline Fatigue & $240(36.2 \%)$ & $199(37.1 \%)$ & $41(32.5 \%)$ & .342 \\
\hline Chest pain & $116(17.5 \%)$ & $98(18.2 \%)$ & $18(14.3 \%)$ & .292 \\
\hline Sweating & $56(8.4 \%)$ & $42(7.8 \%)$ & $14(11.2 \%)$ & .232 \\
\hline Anorexia & 152 (22.9\%) & $117(21.8 \%)$ & $35(27.8 \%)$ & .150 \\
\hline Headache & $62(9.4 \%)$ & $53(9.9 \%)$ & $9(7.1 \%)$ & .344 \\
\hline Sore throat & $10(1.5 \%)$ & $9(1.7 \%)$ & $1(0.8 \%)$ & .697 \\
\hline Diarrhea & $40(6.0 \%)$ & $34(6.3 \%)$ & $6(4.8 \%)$ & .505 \\
\hline Nausea/vomiting & 123 (18.6\%) & $103(19.2 \%)$ & $20(15.9 \%)$ & .390 \\
\hline Abdominal pain & $53(8.0 \%)$ & $42(7.8 \%)$ & $11(8.7 \%)$ & .735 \\
\hline Dizziness & $29(4.4 \%)$ & $23(4.3 \%)$ & $6(4.8 \%)$ & .813 \\
\hline Convulsion & $10(1.5 \%)$ & $4(0.7 \%)$ & $6(4.8 \%)$ & .005 \\
\hline Loss of consciousness & $49(7.4 \%)$ & $24(4.5 \%)$ & 25 (19.7\%) & $<.001$ \\
\hline Others & $79(11.9 \%)$ & $58(10.8 \%)$ & $21(16.7 \%)$ & .067 \\
\hline \multicolumn{5}{|l|}{ Vital signs } \\
\hline Pulse rate & $89.01 \pm 15.16$ & $87.76 \pm 13.43$ & $94.30 \pm 20.21$ & .001 \\
\hline Tachycardia & $100(15.5 \%)$ & $68(13.1 \%)$ & $32(26.0 \%)$ & $<.001$ \\
\hline Respiratory rate & $19.24 \pm 4.40$ & $18.89 \pm 4.04$ & $20.67 \pm 5.45$ & .002 \\
\hline Tachypnea & $119(20.7 \%)$ & $82(17.7 \%)$ & 37 (33.3\%) & $<.001$ \\
\hline SBP & $118.57 \pm 18.10$ & $119.18 \pm 17.47$ & $116.00 \pm 20.40$ & .108 \\
\hline DBP & $74.54 \pm 10.71$ & $74.92 \pm 10.26$ & $72.98 \pm 12.36$ & .105 \\
\hline Temperature & $37.47 \pm 0.89$ & $37.45 \pm 0.89$ & $37.56 \pm 0.90$ & .239 \\
\hline $\mathrm{T} \geq 38.5(\%)$ & $108(17.0 \%)$ & $84(16.3 \%)$ & $24(20.2 \%)$ & .309 \\
\hline Oxygen saturation & $89.84 \pm 7.40$ & $91.36 \pm 5.46$ & $83.87 \pm 10.44$ & $<.001$ \\
\hline $\mathrm{SpO} 2 \geq 95$ & 147 (24.8\%) & $139(29.4 \%)$ & $8(6.7 \%)$ & \\
\hline Sp02 (90-94) & $246(41.5 \%)$ & $214(45.2 \%)$ & $32(26.7 \%)$ & $<.001$ \\
\hline Sp02 $<90$ & $200(33.7 \%)$ & $120(25.4 \%)$ & $80(66.7 \%)$ & \\
\hline
\end{tabular}

Values are expressed as mean \pm SD or numbers (\%).

Sp02: Oxygen saturation by pulse oximetry, SBP: Systolic blood pressure, DBP: Diastolic blood pressure. 
Table 3) Baseline laboratory test results of hospitalized COVID-19 patients.

\begin{tabular}{|c|c|c|c|c|}
\hline & $\begin{array}{l}\text { All Patients } \\
\quad(n=684)\end{array}$ & $\begin{array}{c}\text { Survivors } \\
(n=557)\end{array}$ & $\begin{array}{c}\text { Non-Survivors } \\
(n=127)\end{array}$ & P-Value \\
\hline Leukocytes $\left(\times 10^{9} / \mathrm{L}\right)$ & $6.60(4.95-9.35)$ & $6.40(4.90-8.70)$ & $7.80(5.60-11.50)$ & $<.001$ \\
\hline Increased, $>11.20 \times 10^{9} / \mathrm{L}$ & $105(16.0 \%)$ & $70(13.2 \%)$ & $35(27.6 \%)$ & $<.001$ \\
\hline Decreased, $<4.20 \times 10^{9} / \mathrm{L}$ & $87(13.2 \%)$ & $73(13.8 \%)$ & $14(11.0 \%)$ & .412 \\
\hline Neutrophil count $\left(\times 10^{9} / \mathrm{L}\right)$ & $4.66(3.23-7.01)$ & $4.39(3.11-6.49)$ & $6.06(4.19-9.34)$ & $<.001$ \\
\hline Increased, $>7.70 \times 10^{9} / \mathrm{L}$ & $134(20.5 \%)$ & $89(16.9 \%)$ & $45(36.0 \%)$ & $<.001$ \\
\hline Decreased, $<1.50 \times 10^{9} / \mathrm{L}$ & $12(1.8 \%)$ & $10(1.9 \%)$ & $2(1.6 \%)$ & 1.000 \\
\hline Lymphocyte count $\left(\times 10^{9} / \mathrm{L}\right)$ & $1.20(0.87-1.72)$ & $1.27(0.95-1.77)$ & $0.94(0.64-1.31)$ & $<.001$ \\
\hline Increased, $>4.00 \times 10^{9} / \mathrm{L}$ & $10(1.5 \%)$ & $8(1.5 \%)$ & $2(1.5 \%)$ & 1.000 \\
\hline Decreased, $<1.00 \times 10^{9} / \mathrm{L}$ & $221(33.8 \%)$ & $151(28.6 \%)$ & $70(56.0 \%)$ & $<.001$ \\
\hline Platelets $\left(\times 10^{9} / \mathrm{L}\right)$ & $183.0(145.0-237.0)$ & $186.0(146.8-234.0)$ & $168.0(135.0-246.0)$ & .163 \\
\hline Increased, > $450.0 \times 10^{9} / \mathrm{L}$ & $15(2.3 \%)$ & $11(2.1 \%)$ & $4(3.1 \%)$ & .506 \\
\hline Decreased, $<150.0 \times 10^{9} / \mathrm{L}$ & $177(26.9 \%)$ & $135(25.5 \%)$ & $42(33.1 \%)$ & .083 \\
\hline Hemoglobin (g/dL) & $12.8(11.5-13.9)$ & $12.9(11.7-14.0)$ & $12.3(10.6-13.5)$ & .003 \\
\hline Decreased, $<12 \mathrm{~g} / \mathrm{dL}$ & $212(32.3 \%)$ & $158(29.8 \%)$ & $54(42.5 \%)$ & .006 \\
\hline CRP (mg/L) & $38.8(16.7-68.4)$ & $33.5(15.4-63.0)$ & $53.7(28.8-89.0)$ & $<.001$ \\
\hline Increased, > $10 \mathrm{mg} / \mathrm{L}$ & $519(84.9 \%)$ & $405(83.0 \%)$ & $114(92.7 \%)$ & .007 \\
\hline $\operatorname{ESR}(\mathrm{mm} / \mathrm{h})$ & $46.70 \pm 26.29$ & $45.56 \pm 25.37$ & $50.63 \pm 29.04$ & .100 \\
\hline Increased, > $20 \mathrm{~mm} / \mathrm{h}$ & $334(79.9 \%)$ & $261(80.6 \%)$ & $73(77.7 \%)$ & .537 \\
\hline INR & $1.20 \pm 0.46$ & $1.14 \pm 0.31$ & $1.40 \pm 0.73$ & .001 \\
\hline Increased, > 1.26 & $82(19.7 \%)$ & $42(13.4 \%)$ & $40(38.8 \%)$ & $<.001$ \\
\hline PT (s) & $13.19 \pm 4.78$ & $12.52 \pm 3.15$ & $15.24 \pm 7.58$ & .001 \\
\hline Increased, > $13.6 \mathrm{~s}$ & $88(21.2 \%)$ & $47(15.0 \%)$ & $41(40.2 \%)$ & $<.001$ \\
\hline PTT (s) & $27.64 \pm 10.21$ & $26.49 \pm 8.28$ & $31.13 \pm 14.09$ & .002 \\
\hline Increased, $>40 \mathrm{~s}$ & $20(4.9 \%)$ & $10(3.2 \%)$ & $10(9.8 \%)$ & .014 \\
\hline pH & $7.41 \pm 0.07$ & $7.41 \pm 0.06$ & $7.40 \pm 0.10$ & .096 \\
\hline Increased, > 7.41 & $332(53.5 \%)$ & $270(54.5 \%)$ & $62(49.6 \%)$ & .322 \\
\hline Decreased, $<7.31$ & $39(6.3 \%)$ & $21(4.2 \%)$ & $18(14.5 \%)$ & $<.001$ \\
\hline $\mathrm{PCO}_{2}$ (mmHg) & $43.20 \pm 8.54$ & $43.54 \pm 7.95$ & $41.89 \pm 10.48$ & .102 \\
\hline Increased, > $5.20 \mathrm{mmHg}$ & $65(10.7 \%)$ & $49(10.1 \%)$ & $16(12.9 \%)$ & .376 \\
\hline Decreased, $<40.00 \mathrm{mmHg}$ & $177(30.1 \%)$ & $128(27.2 \%)$ & $49(41.2 \%)$ & .003 \\
\hline
\end{tabular}




\section{Continuation of Table 3}

\begin{tabular}{|c|c|c|c|c|}
\hline & $\begin{array}{l}\text { All Patients } \\
\qquad(n=684)\end{array}$ & $\begin{array}{c}\text { Survivors } \\
(n=557)\end{array}$ & $\begin{array}{l}\text { Non-Survivors } \\
\quad(n=127)\end{array}$ & P-Value \\
\hline $\mathrm{HCO}_{3}(\mathrm{mEq} / \mathrm{L})$ & $26.95 \pm 4.79$ & $27.31 \pm 4.44$ & $25.50 \pm 5.77$ & .001 \\
\hline Increased, $>27.00 \mathrm{mEq} / \mathrm{L}$ & $267(46.7 \%)$ & $223(48.6 \%)$ & $44(38.9 \%)$ & .066 \\
\hline Decreased, $<22.00 \mathrm{mEq} / \mathrm{L}$ & $59(9.7 \%)$ & $38(7.8 \%)$ & $21(17.4 \%)$ & .002 \\
\hline Urea (mg/dl) & $35.9(26.0-51.6)$ & $34.0(24.98-46.0)$ & $47.4(33.0-83.7)$ & $<.001$ \\
\hline Increased, $>45.0 \mathrm{mg} / \mathrm{dl}$ & $204(31.4 \%)$ & $136(26.1 \%)$ & $68(53.5 \%)$ & $<.001$ \\
\hline Serum creatinine (mg/dl) & $1.2(1.0-1.4)$ & $1.1(1.0-1.4)$ & $1.35(1.1-2.0)$ & $<.001$ \\
\hline Increased, > $1.5 \mathrm{mg} / \mathrm{dl}$ & $130(20.0 \%)$ & $82(15.7 \%)$ & $48(38.1 \%)$ & $<.001$ \\
\hline Serum sodium (mmol/L) & $137.07 \pm 4.15$ & $137.19 \pm 3.83$ & $136.60 \pm 5.23$ & .238 \\
\hline Increased, $>146.00 \mathrm{mmol} / \mathrm{L}$ & $5(0.8 \%)$ & $0(0 \%)$ & $5(4.0 \%)$ & $<.001$ \\
\hline Decreased, $<133.00 \mathrm{mmol} / \mathrm{L}$ & $82(13.1 \%)$ & $54(10.8 \%)$ & $28(22.4 \%)$ & .001 \\
\hline Serum potassium (mmol/L) & $4.22 \pm 0.60$ & $4.19 \pm 0.57$ & $4.35 \pm 0.69$ & .007 \\
\hline Increased,$>5.00 \mathrm{mmol} / \mathrm{L}$ & $49(7.8 \%)$ & $31(6.2 \%)$ & $18(14.4 \%)$ & .002 \\
\hline Decreased,$<3.80 \mathrm{mmol} / \mathrm{L}$ & $123(19.7 \%)$ & $100(20.0 \%)$ & $23(18.4 \%)$ & .687 \\
\hline CPK (IU/L) & $115.0(62.0-256.0)$ & $99.0(59.0-214.5)$ & $197.0(115.0-478.5)$ & $<.001$ \\
\hline Increased, > $195.0 \mathrm{IU} / \mathrm{L}$ & $174(31.8 \%)$ & $118(27.0 \%)$ & $56(50.9 \%)$ & $<.001$ \\
\hline $\operatorname{AST}(U / L)$ & $34.0(23.0-46.1)$ & $32.0(22.0-43.38)$ & $43.6(29.0-65.0)$ & $<.001$ \\
\hline Increased, > 40.0 U/L & $140(36.6 \%)$ & $95(31.8 \%)$ & $45(54.2 \%)$ & $<.001$ \\
\hline $\operatorname{ALT}(U / L)$ & $25.0(18.0-41.0)$ & $25.0(18.0-40.0)$ & $26.7(18.0-45.0)$ & .331 \\
\hline Increased, > 50.0 U/L & $61(16.1 \%)$ & $44(14.9 \%)$ & $17(20.5 \%)$ & .218 \\
\hline LDH (U/L) & $525.5(428.25-706.75)$ & $507.0(417.0-634.0)$ & $692.0(494.0-915.0)$ & $<.001$ \\
\hline Increased, $>460.0 \mathrm{U} / \mathrm{L}$ & $170(66.4 \%)$ & $119(61.7 \%)$ & $51(81.0 \%)$ & .005 \\
\hline
\end{tabular}

Values are expressed as mean \pm SD or median (25-75 inter-quartile range or numbers $(\%)$.

CRP: C-reactive protein, ESR: Erythrocyte sedimentation rate, INR: International normalized ratio, PT: Prothrombin time, PTT: Partial thromboplastin time, $\mathrm{PCO}_{2}$ : Partial pressure of carbon dioxide, CPK: Creatine phosphokinase, AST: Aspartate aminotransferase, ALT: Alanine aminotransferase, LDH: Lactate dehydrogenase.

number of cases with decreased $\mathrm{PCO}_{2}$ levels was higher among non-survivors $(41.2 \%$ vs. $27.2 \%, p=.003$ ).

Procedures and outcomes: One hundred eighteen patients (17.7\%) were admitted to the intensive care unit (ICU) and received intensive care based on clinical judgment and the disease severity. ICU admission was substantially higher in non-survivors compared with survivors (70.9\% vs. 5.2\%, $p<.001)$. The averagelength of hospitalization was $6.0 \pm 5.6$, which was not significantly different between the two groups. Oxygen support was administered to patients based on the severity of hypoxemia. The number of patients receiving IMV (invasive mechanical ventilation) (70.9\% vs. $1.9 \%)$ and NIMV (non-invasive mechanical ventilation) (18.1\% vs. $2.8 \%)$ was significantly higher among non-survivors $(p<.001)$; in addition, the number of patients requiring dialysis (19.7\% vs. $3.2 \%)$ and tracheostomy $(2.4 \%$ vs. $0.2 \%$ ) during their hospital stay was also significantly higher among non-survivors. Mortality risk factors: Variables significantly associated with mortality in univariate models 
Table 4) Multivariate analysis of risk factors associated with death in hospitalized COVID-19 patients.

\begin{tabular}{lcc}
\hline & Odds Ratio (95\% CI) & P-Value \\
\hline Number of comorbidities & $1.00[$ reference] & - \\
0 & $2.69(0.88-8.24)$ & .084 \\
1 & $5.40(1.33-21.90)$ & .018 \\
2 & $7.62(1.37-42.34)$ & .020 \\
\hline Loss of consciousness & $4.00(1.27-12.67)$ & .018 \\
\hline Sp02 & $1.00[$ reference] & \\
$\quad \geq 95$ & $2.77(0.71-10.78)$ & .141 \\
$90-94$ & $11.41(3.07-42.47)$ & $<.001$ \\
\hline$<90$ & $3.33(1.59-6.97)$ & .001 \\
\hline Decreased lymphocyte count & $8.21(1.49-45.16)$ & .015 \\
\hline Increased CRP & $4.10(1.92-8.73)$ & $<.001$ \\
\hline Increased CPK & $3.14(1.28-7.70)$ & .013 \\
\hline Increased Urea & $3.16(1.043-9.58)$ & .042 \\
\hline Increased serum potassium & & \\
\hline SpO2: Oxygen saturation by pulse oximetry, CRP: C-reactive protein, CPK: Creatine phosphokinase.
\end{tabular}

were included simultaneously in multivariate logistic regression analysis, and their association with mortality was assessed among the study participants (Table 4). In this analysis, it was shown that $\mathrm{O}_{2}$ saturation less than 90 at admission time was substantially associated with higher odds of mortality (OR=11.41, 95\% CI: 3.07, 42.47, $p<.001$ ) among the study participants. This analysis also revealed a statistically significant association between more than two $(\mathrm{OR}=5.40,95 \% \mathrm{CI}: 1.33,21.90$, $p=.018)$ and three (OR=7.62, 95\% CI: 1.37 , 42.34, $p=.020$ ) underlying comorbidities and death. LOC also had higher odds $(\mathrm{OR}=4.00$, 95\% CI: $1.27,12.67, p=.018$ ) in non-survivors compared with survivors. It was also shown that decreased lymphocyte count $(\mathrm{OR}=3.33$, 95\% CI: 1.59, 6.97, $p=.001$ ) and increased levels of CRP (OR=8.21, 95\% CI: 1.49, 45.16, $p=.015)$, CPK (OR=4.10, 95\% CI: 1.92, 8.73, $p<.001)$, urea (OR=3.14, 95\% CI: $1.28,7.70$, $p=.013)$, and serum potassium (OR=3.16 $95 \%$ CI: $1.04,9.58, p=.042$ ) were predictors of death with higher odds in non-survivors., while age categories, DM, HTN, CVD, dyspnea, convulsion, tachycardia, tachypnea, level of leukocytes, neutrophil count, $\mathrm{Hb}, \mathrm{PCO}_{2}, \mathrm{HCO}_{3}, \mathrm{Cr}$, and serum sodium were not independently associated with mortality in multivariate logistic regression analysis.

\section{Discussion}

This study described demographic, clinical, and laboratory manifestations as well as the risk factors of mortality among 684 COVID-19 confirmed cases admitted to a large referral hospital in Tehran during the first three months of the outbreak. Overall, the majority of patients were middle-aged or older men who suffered from at least one chronic comorbidity. After comparing different characteristics of survivors and non-survivors, multivariable logistic regression analysis revealed that having $\geq 2$ and 3 comorbidities, loss of consciousness as a symptom, a baseline $\mathrm{O}_{2}$ saturation less than 90, lymphopenia, increased CRP, increased $\mathrm{CPK}$, increased urea, and increased serum potassium were positively associated with death among COVID-19 admitted patients. While the case fatality rate (CFR) of COVID-19 in different countries has been widely 
variable, ranging from $0.0 \%$ in Singapore to $28.9 \%$ in Yemen, Iran has a fairly high overall CFR (4.2\%) and is ranked among the top ten countries with the highest mortality rate ${ }^{[13]}$. In this study, the mortality rate among the participants was $18.6 \%$, which was lower than national CFR among hospitalized patients in Iran $\left(24.4 \%{ }^{[14]}\right.$. Discrepancies in observed CFR in different settings could be attributed to some factors, including low detection rates, testing limitations (testing only patients with severe symptoms), patients' diverse demographics, and availability and quality of health care ${ }^{[15]}$. To date, many attempts have been made to find efficient therapeutic interventions (i.e. the use of hydroxychloroquine, antiviral agents, corticosteroids, antibodies, and convalescent plasma transfusion) or create a vaccine to combat SARS-CoV-2 [1618]; however, to implement more efficient strategies for prioritizing recourses allocation, it is necessary to conduct further studies to identify other possible risk factors of mortality and severe outcomes, especially in resource-restricted settings.

Similar to previous studies, this study findings showed that most SARS-CoV-2 infected patients were men (55.8\% males, $44.2 \%$ females) ${ }^{[19,20]}$. A large body of evidence has been published, showing that male gender could be considered as a risk factor for adverse outcomes and mortality in COVID-19 patients ${ }^{[21-23]}$, consistent with the present study showing that the number of men was higher among non-survivors compared to survivors ( $60.6 \%$ vs. $54.8 \%$ ); however, this gender difference was not statistically significant. Some studies have demonstrated that the role of gender on the disease outcomes should be better clarified [9, 24]. Older ages have also been linked to higher mortality rates. A review study based on COVID-19 confirmed cases in the US showed that hospital mortality ranged from less than $5 \%$ among patients $<40$ years old and up to $10.5-30.5 \%$ among those $\geq 65$ years old ${ }^{[19]}$. In line with this finding, in the present study, the prevalence of patients $\geq$ 65 years old was significantly higher in nonsurvivors compared to survivors (65.4\% vs. $34.5 \%, p$-value <.001).

The most common symptoms in participants at admission time were cough, fever, dyspnea, myalgia, and fatigue, which is a typical finding compared to previous reviews ${ }^{[3,16]}$. Some of the less common atypical symptoms were chest pain, nausea/vomiting, and anorexia. While anosmia and altered sense of smell or taste have been reported by others [25-26], these symptoms were not assessed in this study due to incomplete medical history records. Compared to survivors, nonsurvivors were more frequently admitted with dyspnea (63.5\% vs. 50.0\%) and neurological symptoms such as convulsion (4.8\% vs. $0.7 \%$ ) and loss of consciousness (19.7\% vs. $4.5 \%)$. It's worth mentioning that after multivariate regression analysis, loss of consciousness was identified as the only symptom associated with mortality among patients studied (OR $[95 \% \mathrm{CI}]=4.00[1.27$ -12.67]). Neurological symptoms have been described as rare manifestations of COVID-19 disease [27]; however, the present study results alongside other reports from some cases of COVID-19 patients in the United Kingdom with neurological involvements ${ }^{[28]}$, emphasized the importance of neurological complications of SARS-CoV-2 infection. According to the baseline vital signs, higher pulse rate (tachycardia), higher respiratory rate (tachypnea), and lower $\mathrm{O}_{2}$ saturation were more prominent in non-survivors compared to survivors; having hypoxia (with $\mathrm{O}_{2}$ saturation < 90\%) was significantly associated with mortality $(\mathrm{OR}[95 \% \mathrm{CI}]=$ 11.41 [3.07 - 42.47]), these findings are consistent with the findings of previous studies ${ }^{[29-30] .}$ 
Approximately $60-90 \%$ of all hospitalized COVID-19 patients have at least one comorbidity ${ }^{[19]}$. Likewise, based on the present study data, more than $67 \%$ of all patients had one or more comorbidities. However, in the non-survivors group, the prevalence of comorbidities increased to more than $81 \%$. In line with previous reports, the most common comorbidities among the participants were hypertension (33.5\%), diabetes mellitus (27.5\%), and cardiovascular disease (19.6\%), which were significantly more prevalent in the non-survivors group ${ }^{[4]}$. The multivariate logistic regression analysis results revealed that having two or more comorbidities increased the odds of mortality, but not a single comorbidity. Mortality rates among pregnant women infected by SARS$\mathrm{CoV}-2$ have been compared with general population in some studies ${ }^{[31]}$. In this study, there were 10 pregnant women among the participants, all of whom were successfully discharged, and none were in the nonsurvivors group. Regarding obesity and its association with outcomes of COVID-19 patients, while some reports have linked obesity with severe forms of the disease and higher mortality rate ${ }^{[32-33]}$, other studies have failed to establish obesity as a pre-existing condition associated with death ${ }^{[34-35]}$, this finding is in line with the present study results demonstrating the comparable prevalence of obesity and mean BMI between the survivors and nonsurvivors.

The laboratory test results in this study were generally consistent with the findings of previous studies ${ }^{[36-37]}$. Elevated general inflammatory indices, such as leukocytosis and neutrophilia, as well as increased CRP were found to be common in patients but significantly more common in nonsurvivors. An important hematological abnormality observed in COVID-19 patients is lymphopenia ${ }^{[38]}$, which was also observed in participants and mostly in non-survivors. Other laboratory test results which were more prominent among non-survivors included impaired coagulation test (increased PT, PTT and INR), imbalanced venous blood gases (decreased $\mathrm{HCO}_{3}$ and $\mathrm{PCO}_{2}$ ), impaired kidney function (increased serum urea and creatinine), tissue damage indices (increased CPK and LDH), imbalanced serum electrolyte levels (increased serum sodium and potassium), and impaired liver enzymes (increased AST). Several laboratory abnormalities have been reported to be associated with severe outcomes ${ }^{[24,39]}$. The multivariate regression analysis results revealed that five parameters better predicted mortality, including increased CRP, increased CPK, lymphopenia, increased serum potassium, and increased urea (from the highest OR to the lowest, respectively).

This study has some limitations that must be acknowledged. First, the observational retrospective design restricts the accuracy of findings. Second, this study was based on a single-center hospital, which may not broadly reflect the general population of Iran. Third, some missing data were detected in medical documents and laboratory test results. Therefore, some of the laboratory variables were not included in logistic multivariate analysis (in particular AST, PT, PTT, INR and LDH). Lastly, some of pharmacological and therapeutic strategies patients received were not assessed. On the other hand, this study has quiet noteworthy strengths too. For instance, it was one of the first studies reporting epidemiological, clinical, and laboratory characteristics of COVID-19 patients in Tehran; in addition, the sample size was relatively adequate, which allowed us to perform a multivariate logistic regression analysis to better concentrate on predictors of mortality. 


\section{Conclusion}

In conclusion, this study showed that among COVID-19 individuals admitted to hospital, some baseline features could predict morbidity, such as patient's epidemiological background (having more than two or three comorbidities), symptoms (loss of consciousness), signs $\left(\mathrm{O}_{2}\right.$ saturation $<90 \%$ on pulse oximetry), and laboratory test results (increased CRP, CPK, urea, and serum potassium). As SARS-CoV-2 still remains as a major public health challenge, it is necessary to conduct further longitudinal prospective studies designed as case-controls and clinical trials to better understand the risk factors associated with mortality in different populations.

Acknowledgements: The authors would like to thank Imam Hossein hospital staff and patients who took part in this study. They would also like to thank and acknowledge Professor Amir Kavousi for his guidance in statistical analysis and Mrs. Soheila Rahavard who participated in data entry process. This study was the subject of the MPH dissertation of Negar Delbari at School of Public Health and Safety, Shahid Beheshti University of Medical Sciences.

Ethical Permission: This study was approved by Iran National Committee for Ethics in Biomedical Research with the ethical code number: IR.SBMU.PHNS. REC.1399.030.

Conflict of interests: The authors declare no conflicts of interests.

Authors' contribution: Conceptualization: $\mathrm{MH}$; Data curation and formal analysis: SA and ND; Investigation: SA and ND; Methodology and project administration: $\mathrm{HH}$; Supervision: $\mathrm{HH}$ and $\mathrm{MH}$; Validation: $\mathrm{HH}$ and $\mathrm{MH}$; Writing of original draft: $\mathrm{SA}$ and ND; Writing, reviewing, and editing: SA and $\mathrm{ND}$ and $\mathrm{HH}$.

Fundings: The authors did not receive any funding for this study.

Consent to participate: Written informed consents were obtained from participants.

\section{References}

1. Li Q, Guan X, Wu P, Wang X, Zhou L, Tong $Y$, et al. Early transmission dynamics in Wuhan, China, of novel coronavirusinfected pneumonia. New Eng J Med. 2020;382(13):1199-207.

2. World Health Organization. WHO coronavirus disease (COVID-19) dashboard. 2021.

3. Struyf T, Deeks JJ, Dinnes J, Takwoingi Y, Davenport C, Leeflang MM, et al. Signs and symptoms to determine if a patient presenting in primary care or hospital outpatient settings has COVID-19 disease. Cochrane Database Syst Rev. 2020;7(7):Cd013665.

4. Mahumud RA, Kamara JK, Renzaho AMN. The epidemiological burden of and overall distribution of chronic comorbidities in coronavirus disease-2019 among 202,005 infected patients: evidence from a systematic review and meta-analysis. Infection. 2020;48:813-33.

5. Abdi M. Coronavirus disease 2019 (COVID-19) outbreak in Iran: Actions and problems. Infect Control Hosp Epidemiol. 2020;41(6):754-5.

6. Iran Population 2020. World Population Review website; 2020. [cited 29 Aug 2020] Available from: https:// worldpopulationreview.com/countries / iran-population.

7. Arab-Zozani M, Ghoddoosi-Nejad D. Covid-19 in Iran: The good, the bad, and the ugly strategies for preparedness - A report from the field. Disaster Med Public Health Prep. 2020:1-3.

8. COVID-19 coronavirus pandamic. Worldometer website; 2020. [cited 25 Aug 2020] Available from: https:// www. 
worldometers.info/coronavirus/.

9. Javanian M, Bayani M, Shokri M, SadeghiHaddad-Zavareh M, Babazadeh A, Yeganeh B, et al. Clinical and laboratory findings from patients with COVID-19 pneumonia in Babol North of Iran: A retrospective cohort study. Rom J Intern Med. 2020;58(3):161-7.

10. Shahriarirad R, Khodamoradi Z, Erfani A, Hosseinpour H, Ranjbar K, Emami Y, et al. Epidemiological and clinical features of 2019 novel coronavirus diseases (COVID-19) in the South of Iran. BMC Infect Dis. 2020;20(1):427.

11. Akbari A, Emami A, Javanmardi F, Pirbonyeh N, Fadakar N. Early epidemiological analysis of CoVID-19: First report from South of Iran. Res Sq; 2020:1-12.

12. WHO COVID-19 Case definition. WHO website; 2020 [cited 6 Nov 2020] Available from: https://www.who.int/ publications/i/item/WHO-2019-nCoVSurveillance_Case_Definition-2020.1.

13. Hopkins J. Mortality analysis. Johns Hopkins Coronavirus Resource Center; 2020.

14. Jalili $\quad M$, Payandemehr $\quad P$, Saghaei A, Nouri Sari H, Safikhani HR, Kolivand P. Characteristics and mortality of hospitalized patients with COVID-19 in Iran: A national retrospective cohort study. Ann Intern Med. 2021;174(1):12570 .

15. Beeching NJ, Fletcher TE, Fowler R. BMJ best practice - Coronavirus disease 2019 (COVID-19)- Prognosis. London: BMJ Publishing; 2020.

16. Bchetnia M, Girard C, Duchaine C, Laprise C. The outbreak of the novel severe acute respiratory syndrome coronavirus 2 (SARS-CoV-2): A review of the current global status. J Infect Public Health. 2020;13(11):1601-10.

17. Thoguluva Chandrasekar V, Venkatesalu
B. Systematic review and meta-analysis of effectiveness of treatment options against SARS-CoV-2 infection. J Med Virol. 2021;93(2):775-85.

18. Dong Y, Dai T, Wei Y, Zhang L, Zheng M, Zhou F. A systematic review of SARS-CoV-2 vaccine candidates. Signal Transduct Target Ther. 2020;5(1):237.

19. Wiersinga WJ, Rhodes A, Cheng AC, Peacock SJ, Prescott HC. Pathophysiology, transmission, diagnosis, and treatment of coronavirus disease 2019 (COVID-19): A Review. Jama. 2020;324(8):782-93.

20. Richardson S, Hirsch JS, Narasimhan M, Crawford JM, McGinn T, Davidson KW, et al. Presenting characteristics, comorbidities, and outcomes among 5700 patients hospitalized with COVID-19 in the New York City area. Jama. 2020;323(20):2052-9.

21. Jin J-M, Bai P, He W, Wu F, Liu X-F, Han $D-M$, et al. Gender differences in patients with COVID-19: Focus on severity and mortality. Front Public Health. 2020;8:152.

22. Sharma G, Volgman AS, Michos ED. Sex differences in mortality from COVID-19 pandemic. Are men vulnerable and women protected? 2020;2(9):1407-10.

23. Parohan M, Yaghoubi S, Seraji A, Javanbakht MH, Sarraf P, Djalali M. Risk factors for mortality in patients with coronavirus disease 2019 (COVID-19) infection: A systematic review and metaanalysis of observational studies. Aging Male. 2020:1-9.

24. Wu C, Chen X, Cai Y, Xia Ja, Zhou X, Xu S, et al. Risk factors associated with acute respiratory distress syndrome and death in patients with coronavirus disease 2019 pneumonia in Wuhan, China. JAMA Intern Med. 2020;180(7):934-43.

25. Wee LE, Chan YFZ, Teo NWY, Cherng BPZ, Thien SY, Wong HM, et al. The role of self-reported olfactory and gustatory 
dysfunction as a screening criterion for suspected COVID-19. Eur Arch OtoRhino-L. 2020;277(8):2389-90.

26. Spinato G, Fabbris C, Polesel J, Cazzador D, Borsetto D, Hopkins C, et al. Alterations in smell or taste in mildly symptomatic outpatients with SARS-CoV-2 infection. Jama. 2020;323(20):2089-90.

27. Ellul MA, Benjamin L, Singh B, Lant S, Michael BD, Easton A, et al. Neurological associations of COVID-19. Lancet Neurol. 2020;19(9):767-83.

28. Paterson RW, Brown RL, Benjamin L, Nortley R, Wiethoff S, Bharucha T, et al. The emergingspectrumofCOVID-19neurology: Clinical, radiological, and laboratory findings. Brain. 2020;143(10):3104-20..

29. Cascella M, Rajnik M, Cuomo A, Dulebohn SC, Di Napoli R. Features, evaluation, and treatment of coronavirus (COVID-19). StatPearls. 2021.

30. Rentsch CT, Kidwai-Khan F, Tate JP, Park LS, King JT, Skanderson M, et al. Covid-19 testing, hospital admission, and intensive care among 2,026,227 United States veterans aged 54-75 years. MedRxiv. 2020.

31. Pettirosso E, Giles M, Cole S, Rees $M$. COVID-19 and pregnancy: A review of clinical characteristics, obstetric outcomes, and vertical transmission. Aust N Z J Obstet Gynaecol. 2020;60(5):640-59.

32. Caussy C, Wallet F, Laville M, Disse E.
Obesity is associated with severe forms of COVID-19. Obesity. 2020;28(7):1175.

33. Simonnet A, Chetboun M, Poissy J, Raverdy V. High prevalence of obesity in severe acute respiratory syndrome coronavirus-2 (SARS-CoV-2) requiring invasive mechanical ventilation. 2020;28(7):1195-9.

34. Dietz W, Santos-Burgoa C. Obesity and its implications for COVID-19 mortality. Obesity. 2020;28(6):1005.

35. Onder G, Rezza G, Brusaferro S. Casefatality rate and characteristics of patients dying in relation to COVID-19 in Italy. Jama. 2020;323(18):1775-6.

36. Beeching NJ, Fletcher TE, Fowler R. BMJ best practice - Coronavirus disease 2019 (COVID-19) - Investigations. London: BMJ Publishing; 2020.

37. Lippi G, South AM, Henry BM. Electrolyte imbalances in patients with severe coronavirus disease 2019 (COVID-19). Ann Clin Biochem. 2020;57(3):262-5.

38. Tavakolpour S, Rakhshandehroo T, Wei EX, Rashidian M. Lymphopenia during the COVID-19 infection: What it shows and what can be learned. Immuno lett. 2020;225:31-2.

39. Tan L, Wang Q Zhang D, Ding J, Huang $\mathrm{Q}$ Tang Y-Q et al. Lymphopenia predicts disease severity of COVID-19: A descriptive and predictive study. Signal Transduct Target Ther. 2020;5(1):33. 